\title{
Perceptions, Awareness, and Attitudes of Primary Health Care Workers Towards Management of Type-2 Diabetes in Jordan: A Qualitative Study
}

\author{
Amer Al-Sahouri ${ }^{1} \&$ Joy Merrell $^{1}$ \\ ${ }^{1}$ College of Human and Health Sciences, Swansea University, UK \\ Correspondence: Amer Al-Sahouri, College of Human and Health Sciences, Swansea University, UK. Tel: \\ 44-749-749-3582.
}

Received: April 8, 2021 Accepted: June 7, 2021 Online Published: June 25, 2021

doi:10.5539/gjhs.v13n8p14 URL: https://doi.org/10.5539/gjhs.v13n8p14

\begin{abstract}
Objective: This study aimed to explore health care workers' perceptions, awareness and attitudes towards the factors that contribute to poor glycemic control among patients with Type-2 diabetes in Jordan.

Material and Methods: A qualitative study was conducted to seek rich, in-depth data about health care workers' perceptions and attitudes regarding Type-2 diabetes. Ten semi-structured interviews were conducted with primary health care workers: four nurses, physician, dietitian, pharmacist, lab technician, dentist and receptionist who were recruited using purposive sampling. All interviews were audio recorded, transcribed and uploaded to the qualitative data analysis software package NVivo 12. Thematic analysis was used to analyze the data.

Results: Four themes were derived from the interview analysis: lack of knowledge of patients regarding diabetes and its management; influence of socio-cultural factors on patients' diabetes management behaviors included eating pattern and physical activity; challenges related to the health care system including medication insecurity and health insurance policies and challenges related to staff shortages and poor patient-staff communication.

Conclusion: The findings suggest that medical and nursing practice could usefully move away from a task-oriented medical model towards a more holistic approach in diabetes management. Health promotion should be delivered in a wider context to target the population in different age groups to raise awareness of diabetes amongst the general population in Jordan. Nurses' skills and knowledge could be utilized to rectify patients' misconceptions and beliefs regarding diabetes treatment.
\end{abstract}

Keywords: attitudes, holistic, Jordan, nursing, perception, promotion, Type-2 diabetes
Abbreviations
BG: Blood Glucose
HCWs: Health Care Workers
IDF: International Diabetes Federation
IFG: Impaired Fasting Glycaemia
MOH: Ministry of Health
PHE: Public Health England

T2DM: Type-2 Diabetes Mellitus

UK: United Kingdome

WHO: World Health Organization

\section{Introduction}

Diabetes mellitus is a significant health problem in Jordan (International Diabetes Federation (IDF), 2017). The prevalence rate of all types of diabetes increased from $13.1 \%$ in 2016 to $23.7 \%$ in 2019 (World Health Organization (WHO), 2016; Ajlouni et al., 2019). Diabetes affects quality of life, lifestyle, mental wellbeing, finance and independence (Hillson, 2015). 
Good glycemic control levels have been shown to decrease rates of diabetes microvascular complications such as retinopathy, nephropathy and neuropathy (IDF, 2017). By contrast, poorly controlled diabetes leads to premature death and significant complications such as cardiovascular, neurological and renal diseases (WHO, 2016).

Diabetes has many negative consequences, which can be prevented or delayed via increased patients' awareness and support provided by health care workers (HCWs) to manage their diabetes effectively (Whicher, O'Neill, \& Holt, 2020). Perception, knowledge and attitudes of HCWs especially nurses are crucial to provide high quality and safe care for patients with diabetes (Priharjo \& Birdsall, 2010). HCWs educate patients to develop appropriate knowledge and experience which enables them to manage their diabetes at home (Lawler, Trevatt, Elliot, \& Leary, 2019). Effective communication between patients and HCWs influences patients' perceptions that may improve patients' understanding of glycemic control levels and diabetes management among patients with poorly controlled diabetes (Bakkar, Haddad, \& Gammoh, 2017).

Poorly controlled diabetes is considered a significant public health problem associated with many negative consequences at all levels in Jordan (Al-Khawaldeh, Al-Hassan, \& Froelicher, 2012; Alhabashneh, Khader, Herra, \& Asa'ad, 2015; Al-Sahouri, Merrell, \& Snelgrove, 2019).

As the prevalence rate of poorly controlled T2DM among Jordanian people with diabetes is high, a qualitative study was needed to gain a better understanding of the factors associated with poor control of diabetes from the perspectives of HCWs who provide direct care for patients with T2DM. The perceptions of patients with poorly controlled Type 2 diabetes in Jordan have previously been reported in the literature.

In Jordan, although most of the people with T2DM receive their diabetes treatment through primary care services, there is a scarcity of published qualitative research about HCWs' perceptions of diabetes in primary health care (Al-Khawaldeh et al., 2012; Al-Sahouri et al., 2019).

This study was conducted in a primary care setting to add to the limited existing body of knowledge regarding the views of primary HCWs in supporting people with T2DM in Jordan. To date, there are no published qualitative studies identified from Jordan regarding primary HCWs' perceptions of the barriers to good glycemic control. The aim of this study was to gain a better understanding of the factors associated with poor control of diabetes from the perspectives HCWs in a primary care setting.

The health care system in Jordan consists of six main sectors: The Ministry of Health, Royal Medical Services, the private sector, public university hospitals, National Centre for Diabetes and the United Nations Relief and Works Agency (Ministry of Health (MOH), 2018). The majority of primary care centers are managed by The Ministry of Health and are accessible to people living with T2DM in rural and urban areas in Jordan (MOH, 2018). This study was undertaken in one of the comprehensive care centers in a large city. The comprehensive health care centers provide general medical services and the main medical specialties such as internal medicine, family medicine, respiratory and infectious diseases along with maternal and child health, dental and public health services (MOH, 2009).

\section{Methods}

This qualitative study was conducted in a comprehensive health care center in urban area in Jordan between July and December 2016. The selected center was a large-sized center in a large city, with a considerable number of people accessing its health care services and facilities. 120,578 patients received primary care services from this center between January and December 2017 of whom 44,651 patients were aged $\geq 45$ years (MOH, 2018). This center provided comprehensive health care services for inhabitants who live in three regions of the city. Inclusion criteria included HCWs who provided formal care to patients with T2DM and who had worked for more than six months in the selected center. This was to ensure that they had knowledge and experience of providing care to patients with T2DM. The average number of HCWs in the selected health care center was 55 people (17 nurses, 8 physicians, 5 dietitians, 7 pharmacists, 7 lab technicians, 4 dentists and 7 receptionists). The total sample size for HCWs was 10 participants. This sample size was considered adequate for addressing the research aim and objectives, although it was acknowledged that the intention was to develop a rich and full understanding of the phenomenon of poorly controlled T2DM in Jordan and that this sample may need to be expanded in light of emerging issues arising from data analysis or until reaching data saturation which is the endpoint in the data collection process when no new material or comments are generated (Bryman, 2016).

To gain different perspectives and views, the researchers conducted interviews with four nurses, physician, dietitian, pharmacist, lab technician, dentist and receptionist. Receptionists were included, as all patients had to visit the reception desk which was considered the first point of contact for patients during their visit to the center. Seeking data from different groups of HCWs provided holistic, rich, in-depth data that facilitated the credibility of 
the findings (Silverman, 2011). The exclusion criteria included HCWs who did not provide care for patients with T2DM.

Though aspects of the case study design were evident in this study as the researchers looked at one comprehensive health care center which could have been considered as one case. However, the focus of this study was not the center per se but the perceptions of the HCWs regarding the factors which facilitated and the barriers which impacted on poor glycemic control. Also, focusing only on a single case study was not the objective of this study. This study aimed to collect data from multiple perspectives and a wide range of participants.

A semi-structured interview was chosen to use in this study. The semi-structured interview compared with a structured interview, allowed for a more in-depth understanding of issues rather than superficial and limited answers (Bryman, 2016) and enabled the generation of rich information focused on the topics related to my study.

A semi-structured interview guide for HCWs were developed based on the aim and objectives of the study, from reviewing the literature and from expert recommendations. The interview guide focused mainly on the factors associated with poor glycemic control among people with T2DM and potential solutions to improve glycemic control levels.

Informed consent was gained before any data collection from HCWs and before commencing the interview. All participants were informed that participation was voluntary and that they had the right to accept or refuse the audio recording of their interviews. Ethical approval was granted from the College of Human and Health Sciences Ethics Committee at Swansea University in the United Kingdom and the Ministry of Health in Jordan. The interviews were audio recorded and transcribed and data analysis was conducted using NVivo version 12 software to analyze the data thematically.

\section{Results}

Twelve interviews were planned, but $2 \mathrm{HCWs}$ declined to participate. Ten semi-structured interviews were conducted with a mix of different HCWs; four nurses, physician, dietitian, pharmacist, lab technician, dentist and receptionist. The average duration of the interviews was 30 minutes. Analysis of the data revealed four main themes: lack of knowledge of patients regarding diabetes and its management; influence of socio-cultural factors on patients' diabetes management behaviors; challenges related to the health care system and challenges related to HCWs and poor patient-staff communication.

\subsection{Lack of knowledge of patients regarding diabetes and its management}

Many HCWs reported misconceptions and lack of knowledge about diabetes and its management among patients that contributed to poor management of their condition.

Three HCWs $(n=3)$ mentioned that poorly controlled diabetes was attributed to a lack of patient's awareness of insulin therapy and the correct way of storing and taking insulin. A response which is typical of all three HCWs was:

Unfortunately, the patient takes insulin for one year, and his blood sugar remains uncontrolled, and we discover later this patient stores insulin wrongly, or he injects himself in the wrong area (Nurse. Interview 7).

Two HCWs also reported patients' fear of initiating insulin therapy and patients considered insulin as a sign of the end stage of diabetes:

When I add insulin based on the guidelines to the treatment plan, most of the patients are afraid of having insulin as end-stage, so I try to educate them. The patient may be worried regarding injections, and he may have a phobia of needles or hypoglycemia (Physician. Interview 1).

People feel terrified when they are switched to take injections. People are afraid of injections. Many patients don't like to be switched from tablets to insulin (Dentist. Interview 8).

Most of the professionally qualified HCWs $(n=7)$ were aware of patients' perceptions of the side effects of medications and common beliefs about medications:

Not following medication regimen is the most common problem here because of wrong beliefs about the bad effects of medications such as sexual problems or metformin may lead to kidney problems and joint pain (Physician. Interview 1).

A dentist also reported patients' experiences with adherence to diabetes medications, and how development of complications induced some patients to be aware of the importance of diabetes management. The patients' beliefs affected their behaviors to engage in healthy activities due to the perceived threat of developing complications: 
Regarding medications, especially tablets, if the patient doesn't know this situation is serious and complications may develop due to uncontrolled blood glucose, the patient will not take tablets. Some patients do not consider diabetes is a very dangerous disease until they got a diabetic foot, then they started to look after themselves (Dentist. Interview 8).

\subsection{Influence of socio-cultural factors on patients' diabetes management behaviors}

Most of the participants talked about the influence of socio-cultural factors on patients' diabetes management behaviors (diet, medications and physical activity).

\section{The social component of food}

Two HCWs talked about patients with diabetes sharing undifferentiated plates of food as a common social activity which mainly resulted from social habits and strong family and social relationships within the Jordanian society:

The patient got invited with other 4 or 5 people or even two persons, and it is unbelievable that the people would make a specific meal for this patient with diabetes. People make food for all people, and the patient is forced to eat this food so either he eats a bit and stops or he eats with people (Dentist. Interview 8).

Four HCWs $(n=4)$ also reported that patients found it difficult to refuse the served food by hosts and were aware of the role of the socio-cultural context in Jordan in patients' adherence to a diet management plan. The quote below from a doctor is typical of the HCWs' responses:

It is shameful if the patient refuses to eat the offered sweets and says he is a diabetic patient so it is culture or host to serve a small plate (Physician. Interview 1).

Three HCWs referred to the generosity of the hosts as lack of awareness of diabetes because of the increased food quantity and insistence on drinking or eating foods and beverages which will exacerbate the condition:

I am sorry but when people serve you carbonated soft beverages which are the biggest disaster for the patient with diabetes for all people. Then they say 'drink this cup it will not kill you' ....you make the patient frustrated for one million years. The patient says 'I cannot drink because I have got diabetes' then people reply the famous words 'this cup will kill you!! It will not kill you...drink it today'...NO, I do not want to drink it (Nurse. Interview 7).

This insistence of hosts to eat and drink and a reluctance on people with diabetes to refuse what is being offered was reported as being challenging in trying to maintain glycemic control.

Social events and occasions are more prominent in the summer months and people in Jordan have a habit of serving large quantities of high sugar foods during social occasions. The physician who was interviewed, reported the impact of the summer season on patients' diet plans:

In the summer season, people consume a lot of grapes and figs, so the blood sugar is not controlled during the summer season. Attending weddings and family occasions are common during summer when people eat a lot of sweets on these occasions (Physician 1. Interview 1).

Besides the social component of food the emotional component of food was also reported by the HCWs as a factor, which influenced adherence to diet plans

\section{The emotional component of food}

Eating habits go beyond calories, nutrition and relieving hunger; food temptation and lack of willpower related to food were reported by over a third of participants $(n=4)$ as barriers to controlling patients' diet especially during social and family gatherings at home.

Feelings of deprivation were reported as being introduced by inappropriate instructions and advice provided by some HCWs. Inconsistency in advice and some HCWs viewing foods as being forbidden were reported by a nurse as being an influencing factor in poor dietary management. The issue of feeling deprived was also reported by a further three HCWs (including a dentist, doctor and dietitian). Responses which are typical of the four responses made follows:

Patient's will and diet, but the patient should not deprive himself of eating starchy food and sweets. For instance, the doctor tells a patient that sugar, rice, potato and bread are forbidden, so everything is forbidden. The patient says 'I can only drink water' so he will not follow the proper diet. For instance, when I ask the patient for a decreasing amount of rice, he will do it, but when I tell the patient that rice is forbidden, he will not listen to me. Another example when I say sugar is forbidden, I should provide alternatives such as artificial sweeteners or fruit sugar for patients who can drink tea without sugar. Artificial sweeteners and fruit sugar provide patients sugary taste and fewer calories (Nurse. Interview 4). 
It is difficult of patients to follow a diet because they see all people eat sweets, sugar and other things but they eat food with no taste and no smell, so patients feel unhappy (Dentist. Interview 8).

Besides social influences on diet, the data indicated that social factors influenced physical activity too.

Social factors influencing physical activity

HCWs reported that lack of an exercise culture in Jordan and social norms affected patients' efforts to engage in physical activity. A nurse talked about the reason behind a lack of exercise by women due to socio-cultural norms in Jordan:

It is difficult telling young or old women to join the gym or exercise outside due to culture so that walking fast for 15 minutes at home is enough to burn calorie (Nurse. Interview 2).

A lab technician reported patients' negative experiences of undertaking outdoor physical activity and living in a society where physical activity is not part of its culture discouraged patients from exercising. A lack of designated areas for exercise and poor urban planning hindered patients to exercise outside. This HCW reported that

There is no available place for exercise and old patients feel embarrassed to exercise outside. If each health club offers low membership fees for a group of patients and the trainer also shows them the appropriate exercises, $80 \%$ of this problem will be solved (Lab technician. Interview 5).

\subsection{Challenges related to the health care system}

Participants reported several challenges related to the health care system and its policies, and the impact of those policies on service provision and patient care.

Impact of health insurance

The national health insurance schemes and medical exemption policies were identified by 5 HCWs as a barrier that hindered adherence to patients' management plan. Medical exemption means that the patient is only able to receive diabetes care at a specific center. A nurse considered this as a limitation because it affected patients' adherence to any treatment plan as indicated in the following extract:

Patient visits the center based on his medical exemption. When there is a shortage of insulin or medications in the center, the patient has to wait until the medications are available in the center (Nurse. Interview 4).

Four participants reported that most of the patients had access to another health care facility or dual insurance, which aided in addressing the medication shortages but resulted in conflicting advice and patient confusion:

Patients visit different facilities. For example, a patient was confused about the right dose of Metformin because he stayed in the hospital for implanting a pacemaker and the cardiologist there wrote different doses than the ones written in the patient's medical records (Nurse, interview 10).

Whilst another nurse reported that:

A patient went to see the cardiologist in the hospital, and the cardiologist changed some of his medications and stopped Isoket retard tablets, so the doctor in the primary care center was surprised due to stopping Isoket (Nurse, Interview 7).

Incomplete patient medical records was another challenge for HCWs which was attributable to patients switching from one health facility to another:

A few patients' records have no laboratory results for the last 6 or 7 months, and patients left them at home (Lab technician. Interview 5).

A few HCWs $(n=3)$ suggested that all the health insurance systems should be joined as one national insurance system rather than patients holding more than one insurance:

I prefer that all the patients with diabetes hold one insurance and visit one specific doctor who patients trust and the doctor should be an expert in this field. I prefer one doctor who knows the patient's case. I was confused and the patient feels confused and distracted from visiting more than one doctor. Every doctor gives you different information (Nurse. Interview 7).

\section{Medication supply problems}

The main challenges regarding medication supply that were discussed by participants were medication shortages and changing medications brands. Multiple trips to the centre for obtaining all written medications in the prescription was mentioned as a challenge related to the management of diabetes as indicated in the following extract: 
Some patients got tired from standing in the queue, and then they do not find the medications and go home (Nurse. Interview 10).

A few of the HCWs reported the impact of the national health sector policies regarding medication supply on the patient's medication profile. Frequent switching from one brand to another based on the available tender contract in the Ministry meant patients did not receive the same brands of medication consistently. These medication supply policies resulted in inconvenience for many patients and HCWs in my study. Any changes in the medication supply system in the Ministry of Health implied changing the existing treatment plan for patients which affected in turn, patient's adherence to their treatment program:

Firstly, the system of medications brands in the Ministry as an example it is not always the same brand available or.....may be available or the alternative one. For instance, this month the patient takes Amaryl (Glimepiride), the next month there is no Amaryl (Glimepiride) available, the patient takes Diamicron (Gliclazide) then you put the patient on the third drug. Based on what is in the tender, the patient should take the type of drug (Pharmacist. Interview 6).

\section{Unavailability of laboratory tests}

Most of the HCWs in this study were aware of the challenges that patients faced in relation to doing laboratory tests and the impact on patients' health outcomes. HbA1c test and other essential laboratory tests such as postprandial blood glucose (BG) test, lipids profile tests and liver enzymes were not available in the selected center:

HbAlc and random blood sugar are unavailable. Patients face challenges such as transportation and multiple trips in doing these tests in the central laboratory. Fasting blood sugar is only available (Dietitian. Interview 3 ).

A nurse reported that patients might not have the desire to go to another laboratory to do one test. This nurse also suggested that patients may not do the test which may negatively affect their treatment plan when they next came to visit the center:

The patient should make two trips for getting the result of HbAlc in another laboratory. Sometimes the doctor only orders HbAlc and patient feels lazy for travelling two trips to do one test (Nurse. Interview 4).

\subsection{Challenges relating $\mathrm{HCWS}$}

Many participants $(n=8)$ reported the impact of different aspects of staff shortages and poor patient-HCWs communication on patients' management plan.

The patients had to stand in a queue waiting to receive their medical records which affected the patient's privacy in treatment. The average number of patients with chronic conditions who attended the reception desk to receive their records was 150 patients per day and this number increased during weekends. Only 2 or 3 reception staff were on duty with one receptionist at each window. In the registry, there were new staff members who needed more training which resulted in delays in providing records for patients and long waiting times:

Honestly, when my duty is working on the patients'files, they don 't stand up. The rest of the staff except one girl are new. So when they are new persons on the windows, and I get a problem to be solved the patient may stay 30 mins or more only in the queue. It is stressful (Receptionist. Interview 9).

Data analysis indicated that HCWs such as the dentist, the receptionist, the pharmacist, the nurses and the laboratory technician had limited roles in providing health education to patients. Nurses do not see the patients once they had received their prescriptions. Lack of health education was mainly attributed to workload and work demands as reported in the following extracts:

The pharmacist gives the medications to the patients without instructions for taking these medications because of work pressure, busy clinics or long waiting list of patients (Nurse. Interview 4).

Frankly, we don't deliver any education because we don't have time... Load of work, work pressure and a long waiting list. Doctors need to see 50 or 60 patients each day. Sometimes it is excellent if we take the medical history (Dentist. Interview 8).

A nurse who had been involved in delivering the educational program wished if the health education is utilized more in the center and how it helps to identify new cases of diabetes:

I wished we deliver more education as we discovered new cases of diabetes during the educational programs, and their HbAlc readings were high (Nurse. Interview 4).

Incomplete medical records was another issue related to staff shortages and high workload as doctors were only 
able to document the basic notes of the patients' visit in their medical records:

One patient had stopped taking alternative medication without written notice in the file (Physician, Interview 1)

A few patients have no laboratory results for 6 or 7 months in their records (Lab technician. Interview 5).

A nurse reported that patients' visits to the center were not as effective as there was long waiting time for patients and wished to spend more time discussing patients' concerns:

We (HCWs) should see and sit with all the patients who visit the center and give them enough time so the patient can talk about his problems. So that is the thing that I wish to happen to all patients here (Nurse. Interview 7).

Half of the HCWs $(n=5)$ interviewed perceived that patients did not like to read written information and they preferred to receive auditory information rather than visual information. A physician presumed that written communication was an ineffective mode of communication:

We don't have posters, and nobody reads in this center. If you give the patient brochures, he will not read, and it will be discarded in the dustbin later (Physician, Interview 1).

In addition, not all nurses valued written information as aiding health education:

Patients do not read the provided brochures by the Ministry of Health (Nurse. Interview 2). The use of fear based messages to seek to change health behaviors of patients was proposed by approximately two-thirds of the HCWs $(n=7)$ interviewed:

When we (HCWs) tell patients about diabetes complications, so they are afraid of having it. A few patients had shown interest when they saw other patients with diabetes complications. Patients showed interest because they were afraid of the development of complications (Dietitian. Interview 3).

I make patients feel fearful by telling them about complications such as numbness in extremities, vision problems, diabetic neuropathy and a decrease in sexual performance (Nurse. Interview 2).

However, this fear based approach did not seem to be effective in light of the high prevalence of poorly controlled T2DM.

\section{Discussion}

This study aimed to identify perceptions, awareness and attitudes of the primary HCWs towards factors associated with poor glycemic control of T2DM in Jordan.

This study's findings identified misconceptions about diabetes and its treatment as it was reported that that insulin was perceived by patients as an indicator of poor glycemic control. Therefore, encouraging patients to make behavior changes to manage their diabetes was challenging which led to patients requiring insulin therapy and posed different challenges.

According to the HCWs, this perception was attributed to patients who used insulin as having their HbA1c greater than 7 and insulin was added as a result of the high blood glucose levels among patients. Patients misunderstood the progressive nature of diabetes, and that they required the use of insulin therapy when non-insulin treatment options are no longer able to control blood glucose (IDF, 2017). These reported patients' perceptions of insulin are consistent with a previous study conducted with 223 patients with T2DM in Jordan that showed the importance of HCWs' role in addressing these misconceptions (Al-Khawaldeh et al., 2012).

HCWs reported examples of common patients' beliefs about medications such as metformin and side effects that hindered their adherence to diabetes management plan. These misconceptions and beliefs about diabetes medications were mentioned in another study in Jordan (Jarab, Mukattash, Al-Azayzih, \& Khdour, 2018). Skills and knowledge of existing HCWs, such as the nurse and dietician who have been trained to deliver the health education sessions could usefully be drawn upon to provide more health education and rectify these misconceptions.

Development of complications induced patients to adhere to and to develop a more internal locus of control. According to the HCWs' accounts, diabetes complications encouraged some patients to change their health behavior and follow the treatment plan. Seeking to support behavior change before complications have developed is the aim of preventative care which is the role of HCWs especially nurses in seeking to empower patients to make changes in their health behavior. HCWs deliver preventive strategies that aim to support people to change their eating habits, lose weight and do more physical activity (Peate, 2018). Early screening and check-up services are essential HCWs' responsibilities to prevent development of diabetes complications and improve patient's quality of life (Diabetes UK, 2019). 
Social and cultural customs surrounding diet shaped patients' food preferences and also contributed to a high BG level among patients with diabetes. Engaging in social relationships usually included increased consumption of sugar-sweetened beverages such as fruit juice and foods that contain high levels of fats and carbohydrates. Therefore, these dietary choices affected the ability of patients to maintain adherence to the recommended diet. Home eating habits and the nutrition knowledge of family carers also influenced the foods that patients consumed. Self-management behaviors may necessitate changing the types of food prepared and consumed in the home, which may affect family routines (Baig, Benitez, Quinn, \& Burnet, 2015). Sharing of food and communal eating also posed a barrier to the diet changes required for patients with diabetes. Therefore, it was difficult for patients to estimate the right portion size of served food which can be useful for them to help manage their BG levels and improve glycemic control (Barnard, Levin, Gloede, \& Flores, 2018). Serving a small plate was perceived by participants as a sign of disrespect and lack of generosity which went against the social norms of hospitality in Jordan. Serving food in abundance to guests was perceived as a central and essential social activity within the Arab culture. In Arab speaking countries, guests are served a lot of food in abundance, and the socialization process usually requires the consumption of traditional meals consisting of rice and meat (Alsairafi, Taylor, Smith, \& Alattar, 2016). Public health campaigns are needed such as through the mass media to raise awareness of the risk factors associated with diabetes to mitigate these social norms and seek to reduce the prevalence of diabetes.

A sense of social obligation also forced hosts to serve food in excess to guests which added a social burden and pressure on patients to eat more food than was recommended. A previous study identified that when Jordanians invite guests to dinner, and as a sign of generosity, $93 \%(\mathrm{n}=4423)$ of 5000 participants offer large portions, and $91 \%$ $(\mathrm{n}=4341)$ of the study participants keep insisting on people having more food (Bawadi et al., 2012). Public health campaigns can be utilized for raising public awareness of the social and emotional needs of people with diabetes, to improve their lives and those at risk of developing diabetes.

In the Jordanian social context, hospitality and food consumption play an important role in the offering and receiving of food which can be understood within the context of the gift relationship (Mauss, 2002). Hospitality was perceived as a sign of honor and gift giving to guests; therefore, guests had to accept these gifts. There were two types of obligation: the obligation to give food to meet the social norms of hospitality in Jordan by hosts and the obligation to receive food by guests which are understood through applying the gift relationship (Mauss, 2002). The findings regarding hospitality and the insistence by hosts that guests consumed large quantities of food is understood by the gift relationship. Hosts had to offer food and guests were committed to consuming it during the social gathering.

HCWs reported that lack of an exercise culture in Jordan and social norms affected patients' efforts to engage in physical activity. Lack of designated areas for exercise, expensive sport facilities and poor urban planning hindered patients to exercise outside. Participants mentioned that some patients lost their motivation and avoided outdoor activities to avoid disdainful comments from people. Social and cultural norms competed with patients' efforts to do outdoor exercise.

HCWs reported how patients especially old and female patients considered the perceptions of what other people would think about their engagement in physical activity. Patients would evaluate not only the advantage of engaging in physical activity but also what others would say about their engagement in physical exercise. Therefore, patients behaved in a way that other people thought was appropriate as a result of normative pressure.

The national health insurance schemes and medical exemption policies were identified by

HCWs as a barrier that hindered adherence to patients' management plan. In the case of a shortage of medications in the center under study, some of the patients did not have the option to visit another center to obtain their medications, so the patients had to make multiple trips to the center to access all medications. Others who could afford it had to pay for the unavailable medications from private pharmacies, which added a financial burden on the patients.

Participants reported that most of the patients who attended the center had access to another health care facility or dual insurance. The Department of Statistics pointed out that a number of patients had two or more insurances which is considered as one of the reasons behind medication wastage and the high national spending on medications mainly for chronic conditions in Jordan (The Higher Health Council, 2019).

The findings suggested that conflicting advice and patient confusion were apparent among patients who had more than one health insurance and visited more than one health facility to obtain their medications or to receive diabetic care. Participants reported that patients' adherence to medications was affected by receiving contradictory advice from different doctors. Incomplete patient medical records was another implication which was attributable to 
patients switching from one health facility to another. Multiple trips to the center for obtaining all written medications in the prescription was mentioned as a challenge related to the management of diabetes. The discontinuity in medications supplies in the center complicated patients' management plan as patients did not have a sufficient supply of the prescribed medications. This finding was supported by another previous Jordanian study that revealed that long waiting times and multiple visits to health facilities resulted in inconvenience for patients and that medication shortages added more financial burden on the patients as they had to do more visits and may purchase medications from private pharmacies (Alefan, Tashman, Mukattash, \& Azzam,2014).

Frequent switching from one brand to another meant patients did not receive the same brands of medication consistently. These medication supply policies resulted in inconvenience for doctors and confusion for patients in adhering to medication regimen. Doctors need to spend more time to explain these changes in the medication supply system to the patients. Multiple trips, availability of the tests and lack of an appointment system were factors that affected doing the laboratory tests among patients with diabetes in the center. Providing essential tests in the center is crucial and it may facilitate monitoring of the outcomes of patients' treatment plan.

The HCWs in the study prioritized meeting the acute needs of patients due to time pressures and workload, and health education was viewed as an optional extra if time permitted. In Jordan, the focus on patient-centered pharmaceutical care is limited among pharmacists. Medication preparation and dispensing and inventory control are the main duties of most of the pharmacists in Jordan (Nazer \& Tuffaha, 2017). Therefore, the role of pharmacists in health education and promotion is not utilized effectively as it is in other countries such as the UK where the role of pharmacists in preventative care is being extended (Public Health England (PHE), 2019). Also, the lack of appropriate involvement of both nurses and pharmacists in patient-centered team-based care increases the burden on the physicians and may lead to inadequate health education provided to patients with diabetes (PHE, 2019). A study has identified that registered nurses demonstrated a knowledge deficit in clinical and theoretical-based topics, such as initial treatment of hypoglycemia, insulin storage and preparation; meal planning and duration of action with hypoglycemic agents in Jordan. Yacoub et al., 2014). The previous educational programs in the center helped nurses and doctors to discover undiagnosed cases of diabetes among people in the community. Also, to increase patients' awareness, the educational programs was utilized to conduct diabetes screening among participants. In the Jordanian literature, a cross-sectional study was conducted to identify the prevalence of diabetes and impaired fasting glycaemia (IFG) with 1121 people aged 25 years and above found that 49 participants had undiagnosed diabetes and 91 had IFG (Ajlouni et al., 2008). This means that conducting research in the community helped to discover new cases of diabetes.

Nurses in Jordan base their approach to care primarily on the medical model. The dominant method of delivering nursing care is task-oriented, and the focus on patient-centered care is also limited in Jordan (Zahran, 2011). Additionally, the functional nursing approach or task focused care is a common method of nursing care organization in the public sector in Jordan which may result in a fragmentation of patient's care and limited holistic care of patients (Shuriquie, While, \& Fitzpatrick, 2008). The functional models of nursing care reflect a view of nursing as a broad set of divided functions that can be carried out by multiple nurses (Dubois et al., 2013). This also explains how the medical model shapes nursing practice in Jordan and hinders application of the patient-centered care. A patient-centered approach means that care plans should be designed to meet individual's preferences (Dougherty \& Lister, 2015). It empowers patients to make decisions and share responsibilities with health care professionals with support from their families (Dougherty \& Lister, 2015).

HCWs, especially nurses in this study, wished to spend more time discussing patients' concerns but were constrained from doing so because of organizational factors. The task-list system and task-focused care can make it difficult to adjust workloads when necessary by postponing or omitting less essential tasks (Menzies, 1960).

A study of the nature and effect of the anxiety level of nurses in the health care settings identified that the nurses who provided care for a large number of patients were only able to perform a few tasks and had restricted contact with patients (Menzies, 1960. The nurses focused only on performing the important tasks and to reduce the less important ones, which were considered talking with patients due to the pressure of work (Menzies, 1960). This aids understanding of what happens when there is a high workload and task allocation in that nurses are perceived as being very busy, they do not seek to engage with patients to elicit questions, and patients often do not ask the question as they can see the nurses are very busy. This also leads to each patient being cared for by a number of different staff which impedes the development of trusting relationships (Menzies, 1960). Providing only visible psychomotor care without engagement with the patients has been criticized (Menzies, 1960).

Work demands and staff shortages, especially of doctors, affected communication between patients and HCWs. The doctors were seeing on busy days 50 or 60 patients each, so they did not have enough time to encourage 
questioning from patients. Each doctor had to provide care for 30 to 50 patients with chronic conditions in an average clinic day (5 hours). Globally, the lack of primary care physicians and time constraint are barriers to effectively addressing the multiple complex problems presented by patients in primary care settings (Qidwai, Ashfaq, Khoja, \& Merchant, 2011).

Fear-based approach was used to alert patients to the possibility of developing long term complications. The aim of this approach was to encourage patients to perceive that diabetes is a serious condition and the consequences of poorly controlled diabetes are significant enough to adhere to the management plan. However, using this approach by focusing only on the complications of diabetes may be not effective in preventing or at least delaying the development of complications. For instance, based on the chronic conditions care indicators in the center derived from chronic conditions datasheet, $55 \%(\mathrm{n}=162)$ of 295 patients with diabetes had their HbAlc greater than $7 \%$ between January and August 2017.

\subsection{Implications for nursing and health care practice}

1) The skills and knowledge of existing HCWs, such as the nurse and dietician who have been trained to deliver the health education sessions could usefully be drawn upon to provide more health education.

2) HCWs' skills and knowledge could be utilized to rectify patients' misconceptions and beliefs regarding insulin and diabetes treatment.

3) A holistic approach incorporating patient-centered care could usefully be incorporated into educational programs to improve the understanding of patients' health and information needs.

4) The center policies could usefully be reviewed to give the patients the opportunity to see the same doctor as they may feel comfortable and safe being treated by a familiar doctor who also knows them

5) 5. The medical and nursing practice could usefully move away from a task-oriented medical model towards a more holistic approach in diabetes management

\subsection{Limitation of the Study}

The sample size $(\mathrm{n}=10)$ was small and only four nurses, physician, dietitian, pharmacist, lab technician, dentist and receptionist participated so the findings are of direct relevance to the study setting. However, the study findings may be transferred theoretically to other HCWs in similar settings in Jordan. Future research is recommended to include a larger study involving more health care centers in Jordan.

\section{Conclusion}

The study is one of the first to have explored HCWs' perceptions, awareness and attitudes towards the factors that contribute to poor glycemic control among patients with T2DM in Jordan. HCWs face a number of challenges to address the barriers to good glycemic control among patients with T2DM and to meeting the individual and holistic needs of all patients. The findings will help HCWs to identify the barriers and modify existing policies and practices to better meet the needs of patients. There was a lack of patient-HCW communication in respect to health education mainly due to staff workload and time pressures and most of the HCWs were not involved in health promotion initiatives. These findings can aid developments in HCWs' and nursing practice by seeking to value health promotion and its importance to enhance patients' self-management of diabetes. The nursing practice could usefully move away from a task-oriented medical model towards a more holistic and patient-centered approach in diabetes management.

\section{Acknowledgments}

I would like to thank my supportive PhD supervisor Doctor Sherrill Snelgrove for offering supervision, granting me the time, and sustained interest throughout my $\mathrm{PhD}$ journey.

\section{Competing Interests Statement}

The authors declare no conflicts of interest regarding the publication of this paper.

\section{References}

Ajlouni, K., Khader, Y. S., Batieha, A., Ajlouni, H., \& El-Khateeb, M. (2008). An increase in prevalence of diabetes mellitus in Jordan over 10 years. Journal of Diabetes and Its Complications, 22(5), 317-324. https://doi.org/10.1016/j.jdiacomp.2007.01.004

Ajlouni, K., Batieha, A., Jaddou, H., Khader, Y., Abdo, N., El-Khateeb, M., ... \& Al-Louzi, D. (2019). Time trends in diabetes mellitus in Jordan between 1994 and 2017. Diabetic Medicine, 36(9), 1176-1182/ https://doi.org/10.1111/dme.13894 
Alefan, Q., Tashman, K., Mukattash, T., \& Azzam, S. (2014). Medicines shortages in MOH hospitals in Jordan: Problems \& solutions. European Journal of Hospital Pharmacy, 21(Suppl 1). https://doi.org/10.1136/ejhpharm-2013-000436.453

Alhabashneh, R., Khader, Y., Herra, Z., \& Asa'Ad, F. (2015). The association between periodontal disease and metabolic syndrome among outpatients with diabetes in Jordan. J Diabetes Metab Disord, 14(1). https://doi.org/10.1186/s40200-015-0207-5

Al-Khawaldeh, O., Al-Hassan, M., \& Froelicher, E. (2012). Self-efficacy, self-management, and glycemic control in adults with type 2 diabetes mellitus. $J$ Diabetes Complications, 26(1), 10-16. https://doi.org/10.1016/j.jdiacomp.2011.11.002

Al-Sahouri, A., Merrell, J., \& Snelgrove, S. (2019). Attitudes, knowledge, and perceptions of patients regarding type-2 diabetes in Jordan. J Diabetes Mellit, 9, 1-13. https://doi.org/10.4236/jdm.2019.91001

Alsairafi, Z., Taylor, K., Smith, F., \& Alattar, A. (2016). Patients' management of type 2 diabetes in Middle Eastern countries: Review of studies. Patient Preference and Adherence, 1051. https://doi.org/10.2147/ppa.s104335

Baig, A., Benitez, A., Quinn, M., \& Burnet, D. (2015). Family interventions to improve diabetes outcomes for adults. Annals of the New York Academy of Sciences, 1353(1), 89-112.https://doi.org/10.1111/nyas.1284

Bakkar, M., Haddad, M., \& Gammoh, Y. (2017). Awareness of diabetic retinopathy among patients with type 2 diabetes mellitus in Jordan. Diabetes Metab Syndr Obes, 10, 435-441. https://doi.org/10.2147/DMSO.S140841

Barnard, N., Levin, S., Gloede, L., \& Flores, R. (2018). Turning the Waiting Room into a Classroom: Weekly Classes Using a Vegan or a Portion-Controlled Eating Plan Improve Diabetes Control in a Randomized Translational Study. Journal of the Academy of Nutrition and Dietetics, 118(6), 1072-1079. https://doi.org/10.1016/j.jand.2017.11.017

Bawadi, H., Al-Hamdan, Z., Bawadi, H., Ershidat, O., Hammad, F., \& Agraib, L. (2012). Cultural Eating Practices among Jordanians. Food and Nutrition Sciences, 03(06), 790-795. https://doi.org/10.4236/fns.2012.36106

Bryman, A. (2016). Social research methods (5th ed.). Oxford: Oxford University Press.

Diabetes, UK. (2019). The state of the nation 2019, a review of diabetes services in Wales. Retrieved from https://www.diabetes.org.uk/resources-s3/public/2020-06/SOTN\%20report\%20ENGLISH\%5BW\%5D_0.pd f?WQLn4oGFIPmQi3f_SQjptcKkAelHTXdn

Dougherty, L., \& Lister, S. E. (2015). The Royal Marsden manual of clinical nursing procedures $\left(9^{\text {th }}\right.$ ed.). Chichester, West Sussex: John Wiley \& Sons Inc.

Dubois, C., Damour, D., Tchouaket, E., Clarke, S., Rivard, M., \& Blais, R. (2013). Associations of patient safety outcomes with models of nursing care organization at unit level in hospitals. International Journal for Quality in Health Care, 25(2), 110-117. https://doi.org/10.1093/intqhe/mzt019

Hillson, R. (2015). Diabetes care: A practical manual (2nd ed.). Oxford: Oxford University Press.

IDF. (2017). IDF Diabetes Atlas (8th ed.). http://www.diabetesatlas.org.

Jarab, A., Mukattash, T., Al-Azayzih, A., \& Khdour, M. (2018). A Focus Group Study of Patient's Perspective and Experiences of Type 2 Diabetes and Its Management in Jordan. Saudi Pharmaceutical Journal, 26, 301-305. https://doi.org/10.1016/j.jsps.2018.01.013

Lawler, J., Trevatt, P., Elliot, C., \& Leary, A. (2019). Does the Diabetes Specialist Nursing workforce impact the experiences and outcomes of people with diabetes? A hermeneutic review of the evidence. Human resources for health, 17(1), 65. https://doi.org/10.1186/s12960-019-0401-5

Mauss, M. (2002). The gift: The form and reason for exchange in archaic societies. London: Routledge.

Menzies, I. (1960). A Case-Study in the Functioning of Social Systems as a Defense against Anxiety. Human Relations, 13(2), 95-121. https://doi.org/ 10.1177/001872676001300201

Ministry of Health. (2009). Initial report for Primary Health care services in Jordan. Retrieved May 25, 2016, from http://www.csb.gov.jo/csb/Researchers/CSBReleases/studies/ PDF

Ministry of Health. (2018). Primary Health Care Services. Retrieved August 10, 2018, from http://www.moh.gov.jo/Pages/viewpage.aspx?pageID=233

Nazer, L., \& Tuffaha, H. (2017). Health Care and Pharmacy Practice in Jordan. The Canadian Journal of Hospital 
Pharmacy, 70(2), 150-155. https://doi.org/10.4212/cjhp.v70i2.1649

Peate, I. (2018). Fundamentals of applied pathophysiology: An essential guide for nursing and healthcare ( $3^{\text {rd }}$ ed.). Chichester: Wiley-Blackwell.

Priharjo, R., \& Birdsall, T. (2010). Improving the diabetes knowledge of nurses and unregistered practitioners. Journal of Diabetes Nursing, 14(1), 25-29.

Public Health England. (2019). Pharmacy playing a pivotal role in prevention and public health. Retrieved from: https://publichealthmatters.blog.gov.uk/2019/06/28/pharmacy-playing-a-pivotal-role-in-prevention-and-pub lic-health/

Qidwai, W., Ashfaq, T., Khoja, T., \& Merchant, K. (2011). Equity in Healthcare: Status, Barriers, and Challenges. World Family Medicine Journal /Middle East Journal of Family Medicine, 9(6), 33-38. https://doi.org/10.5742/mejfm.2011.96035

Shuriquie, M., While, A., \& Fitzpatrick, J. (2008). Nursing work in Jordan: An example of nursing work in the Middle East. Journal of Clinical Nursing, 17(8), 999-1010. https://doi.org/10.1111/j.1365-2702.2007.01973

Silverman, D. (2011). Interpreting qualitative data: A guide to the principles of qualitative research (4th ed.). Los Angeles: Sage.

The Higher Health Council. (2019). The National Strategy for Health Sector in Jordan 2015-2019.Retrieved September 15, 2019, from http://www.hhc.gov.jo/uploadedimages/2015-2019.pdf

Whicher, A., O’Neill, S., \& Holt, R. (2020). Diabetes in the UK: 2019. Diabetic Medicine, 37(2), 242-247. https://doi.org/10.1111/dme.14225

World Health Organization. (2016). Global report on diabetes. Geneva: World Health Organization.

Yacoub, M., Demeh, W., Darawad, M., Barr, J., Saleh, A., \& Saleh, M. (2014). An assessment of diabetes-related knowledge among registered nurses working in hospitals in Jordan. International Nursing Review, 61(2), 255-262. https://doi.org/10.1111/inr.12090

Zahran, Z. (2011). Nurse education in Jordan: History and development. International Nursing Review, 59(3), 380-386. https://doi.org/10.1111/j.1466-7657.2011.00947

\section{Copyrights}

Copyright for this article is retained by the author(s), with first publication rights granted to the journal.

This is an open-access article distributed under the terms and conditions of the Creative Commons Attribution license (http://creativecommons.org/licenses/by/4.0/). 